INVESTIGACIÓN

\title{
CONDUCTIVIDAD TÉRMICA DE POLVOS DE ARCILLAS UTILIZADAS EN LA INDUSTRIA CERÁMICA EN OCAÑA NORTE DE SANTANDER Y LA REGIÓN
}

\section{THERMAL CONDUCTIVITY OF CLAY POWDERS USED IN THE CERAMIC INDUSTRY IN OCAÑA NORTE DE SANTANDER AND THE REGION}

\author{
MSc. Ricardo Andrés García León ${ }^{a}$, MSc. Eder Flórez Solano ${ }^{\text {b }}$, MSc. Edwin Espinel Blanco ${ }^{c}$ \\ ${ }^{a}$ Grupo de Investigación en Ingenierías Aplicadas para la Innovación Gestión y Desarrollo \\ "INGAP”. Universidad Francisco de Paula Santander Ocaña. Contacto: ragarcial@ufpso.edu.co \\ ${ }^{\mathrm{b}}$ Grupo de Investigación en Ingenierías Aplicadas para la Innovación Gestión y Desarrollo \\ “INGAP”. Universidad Francisco de Paula Santander Ocaña. Contacto: enflorezs@ ufpso.edu.co \\ ${ }^{c}$ Grupo de Investigación GITYD. Universidad Francisco de Paula Santander Ocaña. Contacto: \\ eeespinelb@ufpso.edu.co
}

Fecha de recepción: 08-04-2017

Fecha de aprobación: 15-06-2017

\begin{abstract}
Resumen: Actualmente en la región Norte de Santander, existen grandes yacimientos de materia prima para la fabricación de productos de mampostería para la construcción y otras industrias, por esta razón que surge la iniciativa de estudiar la capacidad que tiene el polvo de arcilla para conducir calor. En donde posteriormente, se realizó el estudio tecnológico a nivel laboratorio para determinar el valor promedio de conducción de calor de las muestras seleccionadas a distintos voltajes y de esta manera comprobar las características térmicas de las arcillas de la región con referencias bibliografías. Los resultados demostraron la buena capacidad de conducir calor de las muestras de entre 0,09533 y $0,15974 \mathrm{~W} / \mathrm{m}^{\circ} \mathrm{C}$, estando dentro de los rangos determinados en otras investigaciones.
\end{abstract}

Palabras clave: Arcilla, Muestras, Materia prima, Conductividad Térmica, Banco de pruebas.

Abstract: Currently in the North of Santander, there are large deposits of raw material for the manufacture of masonry products for construction and other industries, for this reason the initiative arises to study the ability of clay dust to conduct heat. Afterwards, the technological 
study at the laboratory level was carried out to determine the average value of heat conduction of the selected samples at different voltages and thus to verify the thermal characteristics of the clays of the region with bibliographical references. The results demonstrated the good heat conduction of the samples of between 0,09533 and $0,15974 \mathrm{~W} / \mathrm{m}^{\circ} \mathrm{C}$, being within the ranges determined in other investigations.

Keywords: Clay, Samples, Raw Material, Thermal Conductivity, Test Bench.

\section{INTRODUCCIÓN}

El proceso cerámico se compone principalmente de tres fases, que son; preparación de la pasta cerámica, moldeo de la pieza y cocción. En la primera fase del proceso se modifica la composición y la plasticidad de la arcilla con el fin de obtener una pasta cerámica homogénea, luego se moldea según la forma deseada, presión o extrusión. Una vez, se ha obtenido la pieza moldeada se procede al secado controlando la velocidad de evaporación de agua con el fin de evitar defectos en la producto. Posteriormente, se somete al proceso de cocción con el fin disminuir la porosidad, aumentar la densidad y la resistencia mecánica (Martinez, 2012)(Barranzuela, 2014)(Jacome Manzano, 2015).

El proceso productivo es manejado en tres medidas estratégicas: la explotación minera, la transformación de la arcilla y la comercialización del producto final. Una de las etapas definitivas de este proceso es la cocción, realizada en los hornos. Para su ejecución, el horno pasa por tres etapas: precalentamiento, quema y enfriamiento; deben controlarse en forma correcta, con la finalidad de obtener productos con defectos mínimos y reducir el impacto ambiental (García-León, Flórez Solano, \& Acosta Pérez, 2015).

De acuerdo a su capacidad de producción y desarrollo tecnológico las industrias ladrilleras se han clasificado en chircales, ladrilleras pequeñas, medianas y grandes (Riojas Castillo \& Rodríguez Montaña, 2004).

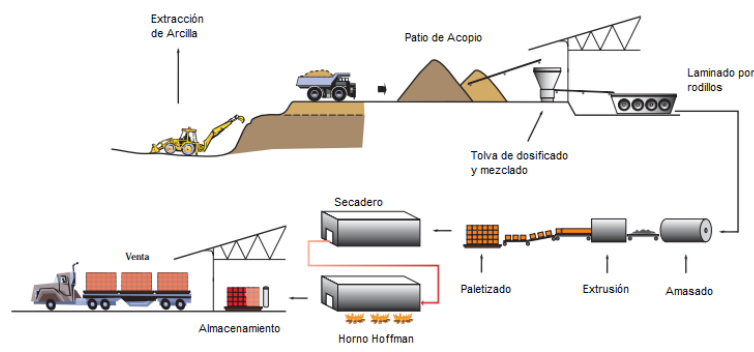

Figura 1. Esquema de fabricación del bloque. Fuente: (T. V. Rodríguez, 2007)

En su mayoría las arcillas se destinan a la industria cerámica de la construcción, un $90 \%$ de la producción se dedica, preferentemente a la fabricación de materiales de construcción y agregados. Sólo un $10 \%$ se dedica a otras industrias (fabricación de papel, caucho, pinturas, absorbentes, decolorantes, arenas de moldeo, productos químicos y farmacéuticos, agricultura, etc.) (Emilia \& Suárez Barrios, 2004).

Estos materiales, son muy usados a nivel de la construcción y otros procesos industriales, pueden ser clasificados atendiendo a multitud de parámetros; así, las minas que contienen minerales arcillosos en su composición pueden ser clasificados dependiendo de su mineralogía, composición química, origen geológico, propiedades físicas, utilización industrial, comportamiento geotécnico, entre otras 
clasificaciones, pero siendo la más común y la más conocida la clasificación mineralógica, entre las que podemos encontrar, según su estructura molecular, arcillas de tipo caolín, illita y montmorillonita (Díaz Rodríguez \& Torrecillas, 2002).

En el área Norte Santandereana existe gran diversidad de productos que se pueden fabricar con esta materia prima, Colombia en estos momentos no es una gran potencia en fabricación industrial de productos a base de arcillas, sin importar que en los suelos exista una gran abundancia de arcillas óptimas. Para el caso de Norte de Santander existe gran variedad de yacimientos naturales; pero a pesar de esto, es un departamento cuya exportación de materiales a base de arcilla no superan el $5,7 \%$ del total de las exportaciones a nivel regional, representando para la economía del departamento aproximadamente 13,7 millones de dólares a Julio de 2013; y teniendo en cuenta que estas cifras, en relación con materiales para la construcción, sólo se ve reflejada para el municipio de Cúcuta y su área metropolitana, pues es el único municipio que posee PYMES en lo referente a fabricación de materiales arcillosos (Fedesarrollo, 2012)(Montoya rivas \& Montoya rivas, 2014).

Generalmente, se utilizan tres componentes que desempeñan papeles importantes en la optimización del rendimiento de las propiedades finales de los bloques y materiales cerámicos. El primer componentes es la arcilla, por lo que su plasticidad facilita la estructura del producto, mientras que el segundo es el feldespato o alúmina $\left(\mathrm{Al}_{2} \mathrm{O}_{3}\right)$ que se utiliza para fundente y la tercera es el sílice $\left(\mathrm{SiO}_{2}\right)$ que se utiliza como un material de relleno y estabilizador (Kamseu et al., 2007)(Monteiro \& Vieira, 2004). Estas composiciones se determinan mediante la análisis químicos, siendo la base de la clasificación modernas de los minerales y la aproximación de las mezclas con los diagramas ternarios (Junkes, Carvalho, Segades, \& Hotza, 2011) (Lassinantti Gualtieri, Romagnoli, \& Gualtieri, 2011).

\section{METODOLOGÍA}

El análisis se le realizo al polvo de arcilla de 5 muestras puras y 2 mezclas de arcillas, las cuales fueron tamizadas en una malla $\mathrm{N}^{\circ} 200$ para escoger aproximadamente 75 gramos. Se siguió el procedimiento establecido en el laboratorio de transferencia de calor de la Universidad Francisco de Paula Santander Ocaña, el equipo funciona aplicando el modelo de cilindro infinito que permite medir la transferencia de calor en la dirección radial y de esta forma definir la capacidad tiene el material de transferir calor al medio, en el cual se compacta por vibración el material granulado de forma que el porcentaje de aire presente en la muestra de la probeta tienda a cero y eliminar en su mayoría la transferencia de calor por convección, para poder determinar de manera más acertada el coeficiente de conductividad térmica presente en el material analizado (Espinel Blanco \& Criado Hernández, 2015) (Cervantes Silva \& Rico Bonilla, 2013) (García-León \& Flórez Solano, 2016)(García-León, Bolívar León, \& Flórez Solano, 2016)(García-León et al., 2015).

Según la información recolectada en libros y artículos, la conductividad térmica de la arcilla varía entre 0,121 y $0,714 \mathrm{~W} / \mathrm{m}{ }^{\circ} \mathrm{C}(\mathrm{G}$. Rodríguez, Moreno, \& Vera, 2008) (Useche Julio, Peña Rodriguez, \& Dulcé Moreno, 2010) (Peña Rodríguez, Peña Quintero, \& Gómez Tovar, 2014). Para las arcillas de estados unidos se considera en la investigación realizada por (Goodhew \& Griffiths, 2005) una conductividad térmica 
de entre 1,3 y $1,53 \mathrm{~W} / \mathrm{m}{ }^{\circ} \mathrm{K}$, en la recopilación de material de transferencia de calor en la universidad de Sevilla, se encontraron con los siguientes valores de conductividad térmica para la arcilla entre 0,49 y $0,87 \mathrm{~W} / \mathrm{m}^{\circ} \mathrm{C}$ (Coronel Toro, 2013), la universidad de las Palmas de Gran Canaria y el libro cengel en su apéndice tiene un valor de conductividad para arcillas secas de 0,93 W/m ${ }^{\circ} \mathrm{K}$ (Cda, 2000) (Cengel, 2007).

La conducción de energía térmica es el mecanismo de Transferencia de Calor en escala atómica a través de los materiales por actividad molecular, donde las partículas con más energía entregan energía a las menos energéticas, produciéndose un flujo de calor desde las temperaturas más altas a las más bajas. La conductividad térmica es una propiedad intrínseca de los materiales que valora la capacidad de conducir el calor a través de ellos. El valor de la conductividad varía en función de la temperatura a la que se encuentra la muestra, este comportamiento está determinado por la ley de Fourier, la cual establece que la tasa de Transferencia de Calor por conducción en una dirección dada, es proporcional al área normal a la dirección del flujo de calor y al gradiente de temperatura en esa dirección. Para un flujo unidimensional de calor se tiene la siguiente ecuación 1:

$$
q_{x}=-A K \frac{\partial T}{\partial X}
$$

Dónde:

qx = Flujo de calor en la dirección $\mathrm{x}$.

$\mathrm{A}=$ Área de la sección transversal.

$\mathrm{k}=$ Constante de proporcionalidad cond. térmica.

$\partial \mathrm{T} / \partial \mathrm{x}=$ Gradiente de temperatura en la dirección $\mathrm{x}$.

Despejando la ecuación 1, se obtiene la ecuación 2 para la conductividad térmica:

$$
-\mathrm{K}=\frac{\mathrm{q}_{\mathrm{x}} * \mathrm{~L}}{\mathrm{~A}\left(\mathrm{~T}_{2}-\mathrm{T}_{1}\right)}
$$

Donde:

$\mathrm{K}=\mathrm{El}$ coeficiente de conductividad térmica.

$\mathrm{q}_{\mathrm{x}}=$ Flujo de calor.

$\mathrm{A}=$ Área normal al flujo de calor.

$\mathrm{T}_{2}$ y $\mathrm{T}_{1}=\mathrm{T}^{\circ}$ medidas en los puntos $1 \mathrm{y} 2$.

Los cálculos se llevan a cabo a partir de las siguientes ecuaciones que rigen la transferencia de calor y de los datos tomados en el análisis.

La ecuación 3, es para calcular la temperatura promedio interior $\left(\mathrm{T}_{\mathrm{i}}\right)$.

$$
\mathrm{T}_{\mathrm{i}=} \frac{\left(\mathrm{T}_{1}+\mathrm{T}_{2}\right)}{2}
$$

La ecuación 4, es para calcular la temperatura promedio exterior $\left(\mathrm{T}_{\mathrm{e}}\right)$.

$$
\mathrm{T}_{\mathrm{e}}=\frac{\left(\mathrm{T}_{3}+\mathrm{T}_{4}\right)}{2}
$$

La ecuación 5, es para calcular la temperatura media $\left(\mathrm{T}_{\mathrm{m}}\right)$

$$
\mathrm{T}_{\mathrm{m}}=\frac{\left(\mathrm{T}_{\mathrm{i}}+\mathrm{T}_{\mathrm{e}}\right)}{2}
$$

Teniendo en cuenta las anteriores ecuaciones, aparece la ecuación 6 para calcular el valor de $\mathrm{K}=$ Conductividad térmica:

$$
\mathrm{K}=\frac{\mathrm{V} * \mathrm{I} * \operatorname{Ln}\left(\frac{\mathrm{r}_{2}}{\mathrm{r}_{1}}\right)}{2 * \pi * \mathrm{~L} *\left(\mathrm{~T}_{\mathrm{i}}-\mathrm{T}_{\mathrm{e}}\right)}
$$

A continuación se presenta la siguiente tabla 1 para encontrar los valores de conductividad térmica:

Tabla 1. Datos para la fórmula de conductividad térmica $\mathrm{K}$.

\begin{tabular}{cccccc} 
& TENSIÓN & & $\mathbf{r}_{\mathbf{1}}$ & $\mathbf{r}_{\mathbf{2}}$ & $\mathbf{L}$ \\
\hline Tensión & Tensión & Tensión & & & \\
$10 \mathrm{~V}$ & $20 \mathrm{~V}$ & $30 \mathrm{~V}$ & 6,7 & 25,4 & $130 \mathrm{~mm}$ \\
Corriente & Corriente & Corriente & $\mathrm{mm}$ & $\mathrm{mm}$ & \\
$175 \mathrm{~A}$ & 350 A & $526 \mathrm{~A}$ & & & \\
\hline Fuente: Elaboración propia & & &
\end{tabular}




\section{RESULTADOS}

Se procedió a calcular cada una de las ecuaciones anteriores para obtener el valor de la conductividad térmica en cada una de las muestras seleccionadas, como se observa las siguientes tablas 2,3 y 4 :

Tabla 2. Datos de conductividad térmica para las 5 muestras a 10 Voltios.

\begin{tabular}{|c|c|c|c|c|}
\hline \multirow[b]{2}{*}{ Muestra } & \multicolumn{4}{|c|}{ TENSIÓN 10} \\
\hline & \multicolumn{2}{|c|}{$\mathbf{T}\left({ }^{\circ} \mathbf{C}\right)$} & & $\frac{\mathbf{w}}{\mathbf{m}-{ }^{\circ} \mathrm{C}}$ \\
\hline \multirow{4}{*}{ M1 } & $\mathrm{Te}=$ & 38,00 & & \\
\hline & $\mathrm{Ti}=$ & 62,63 & $\mathrm{~K}=$ & 0,11595 \\
\hline & $\mathrm{Tm}=$ & 50,31 & & \\
\hline & $\mathrm{Te}=$ & 38,50 & & \\
\hline \multirow{3}{*}{ M2 } & $\mathrm{Ti}=$ & 59,14 & $\mathrm{~K}=$ & 0,13835 \\
\hline & $\mathrm{Tm}=$ & 48,82 & & \\
\hline & $\mathrm{Te}=$ & 41,74 & & \\
\hline \multirow[t]{3}{*}{ M3 } & $\mathrm{Ti}=$ & 66,01 & $\mathrm{~K}=$ & 0,11762 \\
\hline & $\mathrm{Tm}=$ & 53,88 & & \\
\hline & $\mathrm{Te}=$ & 41,75 & & \\
\hline \multirow[t]{3}{*}{ M4 } & $\mathrm{Ti}=$ & 67,04 & $\mathrm{~K}=$ & 0,11291 \\
\hline & $\mathrm{Tm}=$ & 54,39 & & \\
\hline & $\mathrm{Te}=$ & 40,85 & & \\
\hline \multirow[t]{2}{*}{ M5 } & $\mathrm{Ti}=$ & 70,80 & $\mathrm{~K}=$ & 0,09533 \\
\hline & $\mathrm{Tm}=$ & 55,83 & & \\
\hline
\end{tabular}

Fuente: Elaboración propia

Tabla 3. Datos de conductividad térmica para las 5 muestras a 20 Voltios.

\begin{tabular}{|c|c|c|c|c|}
\hline \multirow[b]{2}{*}{ Muestra } & \multicolumn{4}{|c|}{ TENSIÓN 20} \\
\hline & \multicolumn{3}{|c|}{$\mathrm{T}\left({ }^{\circ} \mathrm{C}\right)$} & $\mathbf{w}$ \\
\hline \multirow{4}{*}{ M1 } & $\mathrm{Te}=$ & 74,26 & & \\
\hline & $\mathrm{Ti}=$ & 157,15 & $\mathrm{~K}=$ & 0,13779 \\
\hline & $\mathrm{Tm}=$ & 115,71 & & \\
\hline & $\mathrm{Te}=$ & 79,45 & & \\
\hline \multirow[t]{3}{*}{ M2 } & $\mathrm{Ti}=$ & 169,29 & $K=$ & 0,12713 \\
\hline & $\mathrm{Tm}=$ & 124,37 & & \\
\hline & $\mathrm{Te}=$ & 82,71 & & \\
\hline \multirow{3}{*}{ M3 } & $\mathrm{Ti}=$ & 191,45 & $\mathrm{~K}=$ & 0,10503 \\
\hline & $\mathrm{Tm}=$ & 137,08 & & \\
\hline & $\mathrm{Te}=$ & 81,55 & & \\
\hline \multirow{3}{*}{ M4 } & $\mathrm{Ti}=$ & 182,69 & $\mathrm{~K}=$ & 0,11292 \\
\hline & $\mathrm{Tm}=$ & 132,12 & & \\
\hline & $\mathrm{Te}=$ & 81,63 & & \\
\hline \multirow[t]{2}{*}{ M5 } & $\mathrm{Ti}=$ & 195,00 & $\mathrm{~K}=$ & 0,10074 \\
\hline & $\mathrm{Tm}=$ & 138,31 & & \\
\hline
\end{tabular}

Fuente: Elaboración propia

Tabla 4. Datos de conductividad térmica para las 5 muestras a 30 Voltios.

Muestra

TENSION 30

\begin{tabular}{|c|c|c|c|c|}
\hline & \multicolumn{2}{|c|}{$\mathrm{T}\left({ }^{\circ} \mathrm{C}\right)$} & & $\frac{w}{m-{ }^{\circ} \mathrm{C}}$ \\
\hline & $\mathrm{Te}=$ & 111,45 & & \\
\hline \multirow[t]{3}{*}{ M1 } & $\mathrm{Ti}=$ & 274,46 & $\mathrm{~K}=$ & 0,15794 \\
\hline & $\mathrm{Tm}=$ & 192,96 & & \\
\hline & $\mathrm{Te}=$ & 96,81 & & \\
\hline \multirow[t]{3}{*}{ M2 } & $\mathrm{Ti}=$ & 275,60 & $\mathrm{~K}=$ & 0,14400 \\
\hline & $\mathrm{Tm}=$ & 186,21 & & \\
\hline & $\mathrm{Te}=$ & 126,03 & & \\
\hline \multirow[t]{3}{*}{ M3 } & $\mathrm{Ti}=$ & 322,61 & $K=$ & 0,13096 \\
\hline & $\mathrm{Tm}=$ & 224,32 & & \\
\hline & $\mathrm{Te}=$ & 104,91 & & \\
\hline \multirow[t]{3}{*}{ M4 } & $\mathrm{Ti}=$ & 272,14 & $K=$ & 0,15396 \\
\hline & $\mathrm{Tm}=$ & 188,53 & & \\
\hline & $\mathrm{Te}=$ & 124,75 & & \\
\hline \multirow[t]{2}{*}{ M5 } & $\mathrm{Ti}=$ & 328,61 & $\mathrm{~K}=$ & 0.12629 \\
\hline & $\mathrm{Tm}=$ & 226,68 & & \\
\hline
\end{tabular}

Fuente: Elaboración propia

Teniendo en cuenta lo anterior, resultan las siguientes figuras 2 y 3 que relacionan los resultados obtenidos en el ensayo con datos establecidos teóricamente en función de la temperatura media y la tensión aplicada en el análisis:

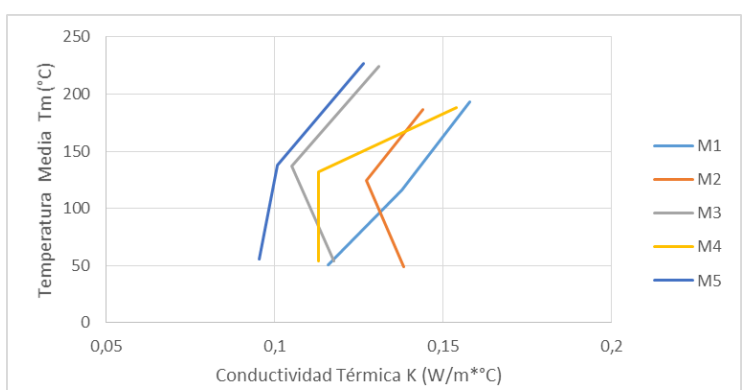

Figura 2. Temperatura media vs conductividad térmica para las 5 muestras.

Fuente: Elaboración propia

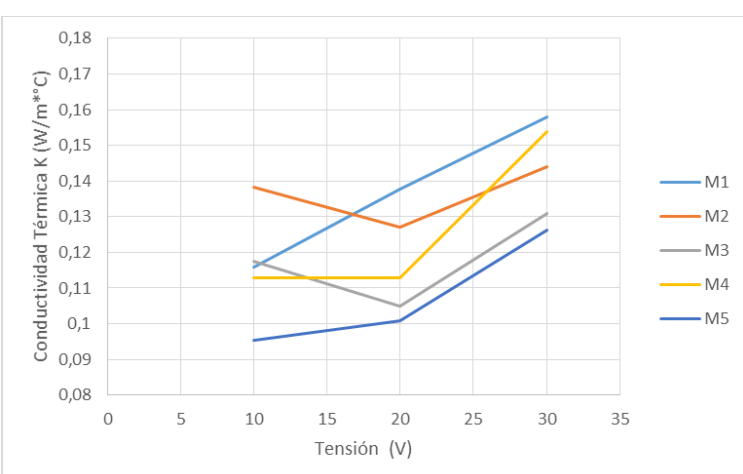

Figura 3. Conductividad térmica vs tensión para las 5 muestras. Fuente: Elaboración propia.

Asimismo, también se realizó el análisis de conductividad a las dos mezclas llamadas 
M7 y M8, de lo que resultaron las siguientes tablas 5, 6 y 7:

Tabla 5. Datos de conductividad térmica para las 2 mezclas a 10 voltios.

\begin{tabular}{clclll}
\hline \multirow{2}{*}{ Muestra } & \multicolumn{3}{c}{ TENSION 10} \\
& & $\mathbf{T}\left({ }^{\circ} \mathbf{C}\right)$ & & $\frac{\mathbf{w}}{\mathbf{m}-{ }^{\circ} \mathrm{C}}$ \\
\hline \multirow{2}{*}{ M7 } & $\mathrm{Te}=$ & 39,11 & & \\
& $\mathrm{Ti}=$ & 64,18 & $\mathrm{~K}=$ & 0,11653 \\
& $\mathrm{Tm}=$ & 51,65 & & \\
\multirow{2}{*}{ M8 } & $\mathrm{Te}=$ & 38,56 & & \\
& $\mathrm{Ti}=$ & 63,41 \\
& $\mathrm{Tm}=$ & 50,99 & $\mathrm{~K}=$ & 0,11626 \\
\hline
\end{tabular}

Fuente: Elaboración propia

Tabla 6. Datos de conductividad térmica para las 2 mezclas a 10 voltios.

\begin{tabular}{|c|c|c|c|c|}
\hline \multirow[b]{2}{*}{ Muestra } & \multicolumn{4}{|c|}{ TENSIÓN 20} \\
\hline & \multicolumn{3}{|l|}{$\mathrm{T}\left({ }^{\circ} \mathbf{C}\right)$} & $\frac{w}{m_{-\circ}^{\circ}}$ \\
\hline \multirow{4}{*}{ M7 } & $\mathrm{Te}=$ & 78,44 & \multirow{3}{*}{$\mathrm{K}=$} & \multirow{3}{*}{0,12187} \\
\hline & $\mathrm{Ti}=$ & 173,80 & & \\
\hline & $\mathrm{Tm}=$ & 126,12 & & \\
\hline & $\mathrm{Te}=$ & 76,36 & \multirow{3}{*}{$\mathrm{K}=$} & \multirow{3}{*}{0,12985} \\
\hline \multirow[t]{2}{*}{ M8 } & $\mathrm{Ti}=$ & 165,50 & & \\
\hline & $\mathrm{Tm}=$ & 120,93 & & \\
\hline
\end{tabular}

Tabla 7. Datos de conductividad térmica para las 2 mezclas a 10 voltios.

\begin{tabular}{clclll}
\multirow{2}{*}{ Muestra } & \multicolumn{2}{c}{ TENSIÓN 30 } \\
& & $\mathbf{T}\left({ }^{\circ} \mathbf{C}\right)$ & & $\frac{\mathbf{w}}{\mathbf{m}-{ }^{\circ} \mathbf{C}}$ \\
\hline \multirow{2}{*}{ M7 } & $\mathrm{Te}=$ & 110,99 & & \\
& $\mathrm{Ti}=$ & 292,86 & $\mathrm{~K}=$ & 0,14273 \\
& $\mathrm{Tm}=$ & 201,93 & & \\
\multirow{2}{*}{ M8 } & $\mathrm{Te}=$ & 111,24 & & \\
& $\mathrm{Ti}=$ & 283,71 \\
& $\mathrm{Tm}=$ & 197,47 & $\mathrm{~K}=$ & 0,15036 \\
\hline
\end{tabular}

Fuente: Elaboración propia

Teniendo en cuenta lo anterior, resultan las siguientes figuras 4 y 5 que relacionan los resultados obtenidos en el ensayo con datos establecidos teóricamente en función de la temperatura media y la tensión aplicada en el análisis:

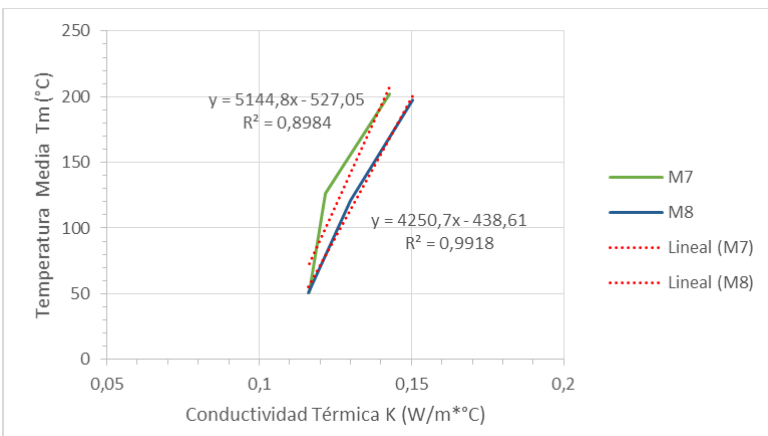

Figura 4. Temperatura media vs conductividad térmica para las 2 muestras.

Fuente: Elaboración propia

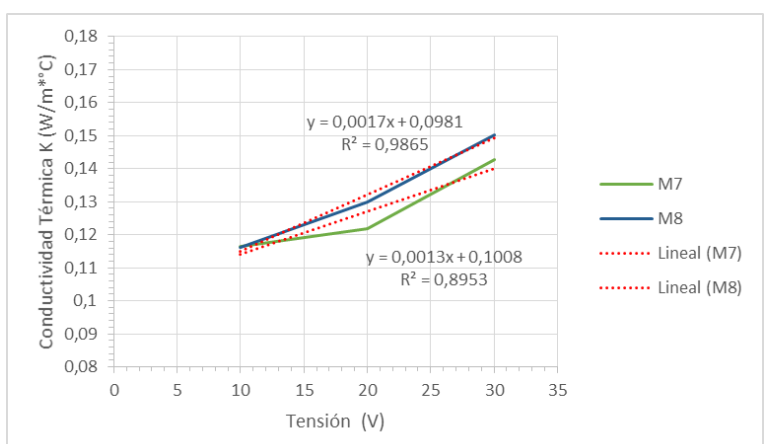

Figura 5. Conductividad térmica vs tensión para las 2 muestras óptimas.

Fuente: Elaboración propia

\section{CONCLUSIONES.}

Como se ve en las gráficas y tablas anteriores, las muestras exhiben una conductividad térmica entre 0,09533 y $0,15974 \mathrm{~W} / \mathrm{m}^{\circ} \mathrm{C}$, por lo que los resultados demuestran que estas muestras de arcilla poseen alta conductividad según lo obtenido en el software sobre materiales, ingeniería, diseño y sostenibilidad.

Al comparar con diferentes materiales, estas muestras tienen una conductividad térmica parecida a las maderas, y algunos polímeros. Sin embargo, su densidad es mucho mayor.

Estos materiales tienen una conductividad térmica parecida, pero la conductividad térmica de ellos no son de materiales que se 
seleccionen precisamente por esta propiedad, en cuyo caso no es posible una futura aplicación por sus características, teniendo en cuenta que los resultados para las mezclas optimas son $0,14273 \mathrm{~W} / \mathrm{m}^{\circ} \mathrm{C}$ y $0,15036 \mathrm{~W} / \mathrm{m}^{\circ} \mathrm{C} \quad$ para $\mathrm{m} 7 \quad \mathrm{y} \quad \mathrm{m} 8$ respectivamente, resultados similares a la investigación realizada por (G. Rodríguez et al., 2008).

Es de vital importancia caracterizar las arcillas químicamente con el objetivo de poder diseñar el producto de acuerdo a la tecnología implementada por la empresa y de esta manera poder optimizar los recursos de la empresa y así los desperdicios generados (García-León \& Bolívar León, 2017).

En trabajos futuros, se establecerá un procedimiento en cuanto a la formulación de la mezcla óptima de arcilla la cual contenga los porcentajes apropiados de material cerámico, con la finalidad de controlar el proceso como también optimizar los recursos.

\section{FINANCIACIÓN}

Los autores agradecen a la División de Investigación y Extensión de la Universidad Francisco de Paula Santander Ocaña, por su apoyo en la financiación de traslados y el desarrollo de pruebas experimentales.

\section{BIBLIOGRAFÍA}

Barranzuela, J. (2014). Proceso productivo de los ladrillos de arcilla producidos en la región piura. Universidad de Piura.

Cda. (2000). Conductividad térmica y densidad de materiales. Universidad de Las Palmas de Gran Canaria, 1-3.

Cengel, Y. (2007). Tansferencia de calor y masa. Un enfoque práctico. Tercera edición. México: McGraw-Hil.

Cervantes Silva, L. F., \& Rico Bonilla, P. (2013). Implementación de un banco de pruebas para determinar la conductividad térmica en materiales granulados en el laboratorio de transferencia de calor de la Universidad Francisco De Paula Santander Ocaña. Colombia.

Coronel Toro, J. (2013). Gráficas y Ecuaciones de Transmisión de Calor (Unviersida, Vol. 3). España. Retrieved from

http://www.esi2.us.es/ jfc/Descargas/T C/Coleccion_tablas_graficas_TC.pdf

Díaz Rodríguez, L. A., \& Torrecillas, R. (2002). Arcillas cerámicas: Una revisión de sus distintos tipos, significados y aplicaciones. Boletin de La Sociedad Espanola de Ceramica $Y$ Vidrio, 41(5), 459-470.

Emilia, M. G. R., \& Suárez Barrios. (2004). Las arcillas (propiedades y usos). Universidad Complutense (Madrid); Universidad de Salamanca, 25.

Espinel Blanco, E., \& Criado Hernández, J. C. (2015). Banco de pruebas para determinar la conductividad térmica como herramienta pedagógica para la enseñanza de transferencia de calor. ACOFI, 8.

Fedesarrollo. (2012). La Mineria en Colombia: Impacto Socioeconómico y Fiscal. Retrieved from http://www.fedesarrollo.org.co/wpcontent/uploads/2011/08/La-mineriaen-Colombia-Informe-de-Fedesarrollo- 
2008.pdf

García-León, R. A., \& Bolívar León, R. (2017). Caracterización Hidrométrica de las Arcillas Utilizadas en la Fabricación de Productos Cerámicos en Ocaña , Norte de Santander. INGE CUC, 13(1), 1-9. http://doi.org/http://dx.doi.org/10.1798 1/ingecuc.13.1.2017.05

García-León, R. A., Bolívar León, R., \& Flórez Solano, E. (2016). Validación de las propiedades físico-mecánicas de bloques H-10 fabricados en Ocaña Norte de Santander y la región. Ingenio UFPSO, 10(2011-642X), 17-26.

García-León, R. A., \& Flórez Solano, E. (2016). Determinación de la ventana del proceso productivo en la fabricación de bloques H-10 en Ocaña Norte de Santander y la región. Ingenio UFPSO, 9(2011-642X), 35-43.

García-León, R. A., Flórez Solano, E., \& Acosta Pérez, M. A. (2015). Análisis estructural de una máquina prensadora para producción de ladrillo macizo para las pequeñas industrias artesanales de materiales cerámicos en Ocaña Norte de Santander y en la región. Revista Colombiana de Tecnologías de Avanzada, 1(1692-7257), 7.

Goodhew, S., \& Griffiths, R. (2005). Sustainable earth walls to meet the building regulations. Energy and Buildings, 37(5), 451-459. http://doi.org/10.1016/j.enbuild.2004.0 8.005

Jacome Manzano, S. A. (2015). Evaluación termodinámica del proceso de cocción $y$ análisis de gases en hornos a cielo abierto y Hoffman en Ocaña. Universidad Francisco de Paula Santander Ocaña.
Junkes, J. A., Carvalho, M. A., Segades, A. M., \& Hotza, D. (2011). Ceramic tile formulations from industrial waste. InterCeram: International Ceramic Review, 60(1), 36-41.

Kamseu, E., Leonelli, C., Boccaccini, D. N., Veronesi, P., Miselli, P., Pellacani, G., \& Melo, U. C. (2007). Characterisation of porcelain compositions using two china clays from Cameroon. Ceramics International, 33(5), 851-857. http://doi.org/10.1016/j.ceramint.2006. 01.025

Lassinantti Gualtieri, M., Romagnoli, M., \& Gualtieri, A. F. (2011). Influence of body composition on the technological properties and mineralogy of stoneware: A DOE and mineralogicalmicrostructural study. Journal of the European Ceramic Society, 31(5), 673685.

http://doi.org/10.1016/j.jeurceramsoc.2 010.12 .002

Martinez, Y. Q. (2012). Caracterización fisicoquímica de cuarzo en el municipio de la playa de belén (Norte de Santander). Universidad Francisco de Paula Santander Ocaña, 84.

Monteiro, S. N., \& Vieira, C. M. F. (2004). Influence of firing temperature on the ceramic properties of clays from Campos dos Goytacazes, Brazil. Applied Clay Science, 27(3-4), 229234.

http://doi.org/10.1016/j.clay.2004.03.00 2

Montoya rivas, G. P., \& Montoya rivas, R. (2014). Caracterización del sector cerámico tradicional del valle de Aburrá y los riesgos profesionales latentes en su proceso productivo. Lámpsakos, 12, 34-42. 
Peña Rodríguez, G., Peña Quintero, J. Y., \& Gómez Tovar, M. A. (2014). Determinación Experimental de la Conductividad Térmica Efectiva en Bloques Extinguidos de Arcilla Roja. Revista Ciencia En Desarrollo, 5(1), $15-20$.

Riojas Castillo, O., \& Rodríguez Montaña, N. E. (2004). Características de hornos para productos cerámicos del Parque Minero Industrial El Mochuelo, localidad 19 de Bogotá, D.C. ConCiencias.

Rodríguez, G., Moreno, J., \& Vera, E. (2008). Conductividad Térmica Efectiva Promedio de Polvos de Arcillas Utilizadas en la Industria Cerámica del Área Metropolitana de San José De Cúcuta. Revista Colombiana de Física, 40(2), 278-280.

Rodríguez, T. V. (2007). Los minerales industriales en Castilla y León. Junta de Castilla y León (Domènech e, Vol. 1). España. http://doi.org/10.1109/EEM.2011.5953 081

Useche Julio, V., Peña Rodriguez, G., \& Dulcé Moreno, J. (2010). Efecto de la presión de compactación en las propiedades termofísicas de polvos de arcilla roja elaboradas por atomización. Respuestas, 2(2), 25-33. 